Revista de Comunicación y Salud, 2021 Vol. 11, 103-123

Editado por Cátedra de Comunicación y Salud

ISSN: 2173-1675

\title{
LA COMUNICACIÓN COMO ELEMENTO FUNDAMENTAL EN LA LITERATURA ESPECIALIZADA EN EXPERIENCIA DE PACIENTE (1989-2013)
}

\section{Communication as a fundamental element of patient experience literature (1989- 2013)}

\author{
Guillem Marca-Francès ${ }^{1}$ \\ Universitat de Vic - Universitat Central de Catalunya. España. \\ quillem.marca@uvic.cat \\ Marc Compte-Pujol \\ Universitat de Vic - Universitat Central de Catalunya. España. \\ marc.compte@uvic.cat \\ Jesica Ana Menéndez-Signorini \\ Universitat de Vic - Universitat Central de Catalunya. España. \\ jesicaana.menendez@uvic.cat \\ Joan Frigola-Reig \\ Universitat de Vic - Universitat Central de Catalunya. España. \\ joan.frigola@uvic.cat
}

\section{Cómo citar el artículo}

Marca-Francès, G., Compte-Pujol, M., Menéndez-Signorini, J. A., y Frigola-Reig, J. (2021) La comunicación como elemento fundamental en la literatura especializada en experiencia de paciente (1989-2013). Revista de Comunicación y Salud, 11, 103123. https://doi.org/10.35669/rcys.2021.11.e269

Financiación. Este trabajo forma parte del proyecto de investigación denominado "Efectividad de una intervención de comunicación para la mejora de la experiencia del paciente crónico durante su estancia en

\footnotetext{
1 Guillem Marca-Francès: Profesor del Departamento de Comunicación, Facultad de Empresa y Comunicación, Universitat de Vic-Universitat Central de Catalunya (UVic-UCC). Miembro del grupo de investigación Learning, Media \& Social Interactions (2017SGR379). Especializado en comunicación de la salud y en relaciones públicas.
} 
el hospital" (CSO2015-71419-R), proyecto competitivo de I+D financiado por el Ministerio de Economía y Competitividad de España.

\title{
Resumen
}

A nivel conceptual, la "experiencia de paciente" comparte varios elementos definitorios con el concepto de comunicación, como la interacción, las relaciones, la información, la reputación o el engagement. En este sentido, el objetivo de nuestro estudio es la identificación de la frecuencia absoluta de la aparición del concepto comunicación y su entorno semántico en 40 artículos representativos del ámbito de la experiencia del paciente previos a la definición de consenso facilitada por The Beryl Institute (2014), así como la asociación del concepto comunicación con resultados clínicos, estableciendo como referencia para la muestra de artículos el trabajo de Doyle, Lennox y Bell (2013). Mediante análisis de contenido, observamos la presencia generalizada de la comunicación de forma directa o indirecta. Concretamente, el término "comunicación" aparece en 29 casos, el término "información" en 25, "relación" en 21 e "interacción" en 21. También se concluye que la comunicación es necesaria para utilizar cualquiera de las herramientas de medición de la experiencia de paciente y que la mayoría de intervenciones en las que interviene la comunicación tiene asociaciones positivas con los resultados clínicos.

Palabras clave: Comunicación de la salud; Experiencia de paciente; Interacciones; Resultados clínicos.

\begin{abstract}
At a conceptual level, "patient experience" shares several defining elements with the concept of communication, such as interaction, relationships, information, reputation or engagement. In this sense, the objective of our study is to identify the absolute frequency of the appearance of the communication concept and its semantic environment in 40 representative articles from the field of patient experience prior to the consensus definition provided by The Beryl Institute (2014), as well as the association of the communication concept with clinical results, establishing the work of Doyle, Lennox and Bell (2013) as a reference for the sample of articles. Through content analysis, we observe the widespread presence of communication directly or indirectly. Specifically, the term "communication" appears in 29 cases, the term "information" in 25, "relationship" in 21 and "interaction" in 21. It is also concluded that communication is necessary to use any of the tools for measuring the patient experience and that most interventions involving communication have positive associations with clinical outcomes.
\end{abstract}

Keywords: Clinical results, Health Communication, Interactions, Patient experience.

\section{INTRODUCCIÓN}

La experiencia del paciente, que ha conquistado el centro de la atención sanitaria en los últimos tiempos (Salomon, 2014), se aleja progresivamente del ámbito de la biomedicina para abarcar un área multidisciplinar en la que interacción, relaciones, información, comunicación, reputación, expectativas o engagement, términos que 
aparecen en distintas definiciones del concepto recogidas por Wolf y otros (2014) y que trasladan el protagonismo a las ciencias sociales.

En esta línea, se tiende a relacionar la capacidad de comunicación de los profesionales asistenciales y no asistenciales de la salud para con los pacientes, con el hecho que estos últimos cumplan las recomendaciones de los médicos, mejoren la autogestión de sus patologías crónicas y adopten conductas preventivas (Institute for Healthcare Communication, 2011). Los estudios realizados durante las últimas tres décadas muestran que la capacidad de los profesionales de la salud para explicar, escuchar y sentir empatía con los pacientes puede tener un profundo efecto en los resultados biológicos y funcionales de su salud, así como en la satisfacción del paciente y la experiencia de la atención.

\subsection{Enfoques de experiencia de paciente}

En sus orígenes, la experiencia de paciente se presentó como una capacidad de respuesta a los pacientes a encontrar en dominios de la autonomía, elección, comunicación, confidencialidad, dignidad, atención inmediata y calidad de los servicios básicos (Bleich, Ozaltin y Murray, 2009).

Para Feirn, Betts y Tribble (2009), la experiencia de paciente se fundamenta en la calidad y el valor de todas las interacciones, directas e indirectas, clínicas y no clínicas, que abarcan toda la duración de la relación paciente/proveedor. Mientras que para Weiss y Tyink (2009), el concepto se basaba en los componentes de una cultura centrada en el paciente que abarca una atención competente y de alta calidad, atención personalizada, respuestas oportunas, coordinación de la atención y una respuesta confiable y receptiva.

Robison (2010) habla de una "experiencia ideal", creada al satisfacer cuatro necesidades emocionales básicas -confianza, integridad, orgullo y pasión-y con la que lograba involucrar a los pacientes. El enlace emocional también lo desarrolla Needham BR. (2012), cuando se refiere a la experiencia del paciente como la administración de una montaña rusa emocional y física que experimenta un paciente mientras se somete a un procedimiento de atención médica y de maximizar la salud y el bienestar social, mental y físico del paciente. Para apoyar este esfuerzo, el autor sugería personalizar la medicina, asociarse con los pacientes y empoderar a los empleados.

Por su parte, Stempniak (2013) marca el inicio y el fin de la experiencia del paciente desde la primera llamada telefónica hasta su alta hospitalaria, e incluye la reducción del sufrimiento y de la ansiedad en todo el continuo de la atención. Mientras que Bowling y otros (2012) se centran en la gestión de las expectativas de los pacientes partiendo de observaciones directas y personales de su atención médica. Unas expectativas que incluyen la limpieza, la información sobre a dónde ir, la citas convenientes y puntuales, el ser atendido a tiempo, la elección del hospital/médico, el personal de recepción, que el médico esté bien informado, sea claro y fácil de entender, que se le involucre en el tratamiento y en la toma de decisiones, que experimente una reducción en los síntomas/problemas de salud, que el médico sea respetuoso y le trate con dignidad, que 
se le dé tranquilidad, que reciba consejos sobre su salud y condición, que se le informe sobre la causa y el manejo de su enfermedad y sobre los beneficios/efectos secundarios del tratamiento, y que tenga la oportunidad de discutir sus problemas.

En la misma línea, Frampton (2012) reduce la experiencia de paciente a dos áreas que contienen las expectativas de Bowling y otros (2012): 1) las interacciones humanas y 2) el ambiente de cuidado. Además, sugiere que la atención centrada en el paciente vive entre la atención y el tratamiento brindados y en cómo los experimentan los pacientes y sus seres queridos.

Pemberton y Richardson (2013) proporcionan una visión de la experiencia del paciente enmarcada por una serie de seis pasos activos, que incluyen: la reputación, la llegada, el contrato, la estancia, el tratamiento y la post-estancia. Los autores también sugieren la importancia de la cultura y el compromiso del personal para impulsar un esfuerzo efectivo de experiencia del paciente, de un modo similar al de Staniszewska y otros (2014), quienes establecen siete temas genéricos clave para lograr una experiencia de paciente de alta calidad: tratar al paciente como un participante activo; la receptividad de los servicios; un enfoque individualizado; la experiencia vivida; la continuidad de la atención; y las relaciones, la comunicación, la información y el apoyo.

Por otra parte, Hewitson y otros (2014) defienden que la experiencia se describe como auto-informes de los pacientes sobre su experiencia de atención hospitalaria, incluidas las interacciones entre el paciente y el profesional, la provisión de información, la participación en las decisiones y el apoyo para el autocuidado y las calificaciones generales de la atención.

Este no es más que un pequeño recopilatorio que finaliza en 2014, coincidiendo con la publicación de la definición de consenso del The Beryl Institute ${ }^{2}$ (2014), que presenta la experiencia del paciente cómo "la suma de todas las interacciones, moldeadas por la cultura de una organización, que influyen en las percepciones del paciente, a lo largo del continuo de la atención". Esta definición contiene cuatro pilares en los que podemos identificar muchos de los elementos planteados por los autores expuestos anteriormente: Interacciones, cultura, percepciones y cuidado continuado.

The Beryl Institute (2014) se refiere con el concepto interacciones introducido en su definición a los puntos de contacto orquestados de personas, procesos, políticas, comunicaciones, acciones y entorno. Feirn, Betts y Tribble (2009) concretan las interacciones en "directas e indirectas, clínicas y no clínicas". Frampton (2012) incluye un adjetivo "interacciones humanas", mientras que Hewitson y otros (2014) especifican las "interacciones entre el paciente y el profesional", pero también concretan "la provisión de información, la participación en las decisiones y el apoyo para el autocuidado" que

\footnotetext{
2 The Beryl Institute es la comunidad de práctica mundial dedicada a mejorar la experiencia del paciente a través de la colaboración y el conocimiento compartido. En 2014 define la experiencia del paciente como la suma de todas las interacciones, conformadas por la cultura de una organización, que influyen en las percepciones del paciente a lo largo de la atención continua.
} 
requieren interacción. Sobre este pilar, y actuando cómo sinónimos de interacción, se sostienen todos los "dominios" (Bleich, Ozaltin y Murray, 2009), la "atención" (Weiss y Tyink, 2009), el "involucrar al paciente" (Robison, 2010) -que requiere interacción-, el "someterse" y "experimentar" (Needham, 2012), el "involucrarse" (Bowling y otros, 2012), los "pasos activos" del paciente (Pemberton y Richardson, 2013), el "paciente activo" y "las relaciones, la comunicación, la información y el apoyo" (Staniszewska y otros, 2014).

La cultura incluida en la definición del The Beryl Institute se refiere a la visión, los valores, las personas (en todos los niveles y en todas las partes de la organización) y la comunidad. Si bien muchas de las definiciones incluyen implícitamente algunos de estos elementos, únicamente dos la incluyen explícitamente: Weiss y Tyink (2009) se refieren a "cultura centrada en el paciente", y Pemberton y Richardson (2013) incluyen la "cultura y el compromiso del personal".

Las percepciones entendidas como lo que reconocen, entienden y recuerdan los pacientes y las personas de apoyo pasan muy desapercibidas en la mayoría de las definiciones. Bowling y otros (2012) introducen el concepto "expectativas", Staniszewska y otros (2014) plantean la experiencia "vivida", y queremos hacer hincapié en el término "vivida" cómo elemento de recuerdo, que va en la misma línea que Hewitson y otros (2014), quienes desde una perspectiva más práctica plantean los "auto-informes de los pacientes sobre su experiencia de atención", incluyendo las "calificaciones generales de la atención".

The Beryl Institute se refiere a los momentos previos, coetáneos y posteriores a la prestación de la atención cuando incluye "cuidado continuado" en su definición. Esta continuidad la presentan de forma explícita Feirn, Betts y Tribble (2009), cuando se refieren a "toda la duración de la relación paciente/proveedor". También Stempniak (2013), cuando habla de "continuo de la atención", o Staniszewska y otros (2014), cuando exponen la "continuidad de la atención". De forma implícita lo encontramos también en las definiciones de Weiss y Tyink (2009), cuando hablan de "coordinación", y en la de Frampton (2012), cuando se refiere a un "ambiente de cuidado".

La lectura de algunas de las definiciones de experiencia de paciente previas a 2014, momento en el que The Beryl Institute establece una base de trabajo hacia el futuro, nos muestra la inclusión de las interacciones por encima de los elementos de cultura, percepción y continuidad. La continuidad es el segundo elemento más presente. El elemento cultural, ya sea desde una perspectiva organizacional o desde una perspectiva personal o social, es clave para el éxito de la experiencia de paciente. No obstante, es el que se muestra más minimizado. La percepción, por su parte, tiende a presentarse como una evaluación o informe de la experiencia vivida, vinculada en algún caso a las calificaciones. Debe tenerse en cuenta que uno de los primeros elementos de contacto entre de los profesionales y las organizaciones proveedoras de servicios de salud con la experiencia de paciente fueron las encuestas de satisfacción HCAPS o Picker, que tienen la finalidad de evaluar la satisfacción de los pacientes entre otros elementos vinculados a la prestación del servicio. 
Tras esta presentación de enfoques de experiencia de paciente ha quedado clara la importancia de las interacciones en casi todas las definiciones presentadas. No obstante, queremos destacar que muy pocas definiciones o abordajes al término incluyen la "comunicación" de forma específica -en concreto, únicamente dos-, mientras que el concepto de "información" aparecería en otros cuatro casos. Además, ninguno de ambos conceptos (ni comunicación, ni información) forma parte de la definición consensuada por The Beryl Institute (2014). Entendemos que la interacción incluida en la experiencia del paciente precisa de comunicación ya sea de tipo personal entre pacientes y profesionales, entre profesionales y profesionales o de carácter organizacional.

Finalmente, cabe destacar que, por los motivos anteriormente mencionados, consideramos 2014 como un punto de inflexión en el abordaje de la experiencia del paciente y, por ese motivo, en el presente artículo abordamos fundamentalmente los elementos previos a esta fecha.

\section{OBJETIVO Y PREGUNTAS DE INVESTIGACIÓN}

Tras revisar en el apartado anterior los distintos enfoques de experiencia de paciente previos al consenso de The Beryl Institute (2014), se observa que la interacción está presente en la mayoría de dichos enfoques. No obstante, la comunicación y la información necesarias para la interacción aparecen únicamente de forma puntual.

Por ello, proponemos como objetivo de este estudio identificar la aparición del concepto comunicación y su entorno semántico en una selección de artículos del ámbito clínico previos a la definición de consenso facilitada por The Beryl Institute (2014). Además, añadimos a esta búsqueda la identificación de una posible asociación del concepto de comunicación con resultados clínicos de los pacientes.

Por coincidencia con las fechas analizadas y por tratarse de un estudio contrastado, se ha considerado establecer como referencia para la muestra de artículos el trabajo de Doyle, Lennox y Bell (2013).

Para llegar al objetivo se desarrollaron 4 preguntas de investigación:

- PI 1 ¿En cuántos estudios se identifica la comunicación como foco de estudio en la experiencia de paciente?

- PI 2 ¿En cuántos estudios aparece la terminología asociada a la comunicación?

- PI 3 ¿Los sistemas de recopilación de datos de experiencia de paciente requieren interacción comunicativa?

- PI 4 ¿En cuántos casos se identifican variables de comunicación asociadas a variables de resultado clínico?

\section{METODOLOGÍA}

Para llevar a cabo los objetivos de investigación, hemos realizado una revisión bibliográfica basada en una selección previa de 55 artículos clave en experiencia de 
paciente, identificados en el trabajo de Doyle, Lennox y Bell (2013). Se ha considerado utilizar la misma muestra debido al rigor metodológico de la selección y a la validación de ésta, al estar el estudio publicado en el BMJ Open.

Los autores construyeron un listado de términos ${ }^{3}$ que abarcaban el campo semántico del concepto experiencia de paciente y los introdujeron en la base de datos EMBASE identificando 5323 artículos. De éstos, analizaron sus resúmenes, hasta seleccionar 18 artículos. Aplicando el método de bola de nieve, ampliaron la muestra a 55. Los criterios de inclusión y exclusión del listado de artículos pretendían identificar estudios originales o revisiones sistemáticas cuyo foco de estudio era la medición de la asociación entre la experiencia del paciente y los resultados de seguridad y efectividad, bien a nivel de paciente o a nivel organizacional, utilizando herramientas validadas y cuyo tamaño de muestra era superior a 50 sujetos (pacientes). En el caso de la medición de la experiencia de paciente las herramientas fueron genéricas -encuesta Picker o HCAPS- o específicas -Working Alliance Scale, Multidimensional Health Locus of Control Scale o Usual Provider Continuity index-.

Sobre la muestra de 55 artículos de Doyle, Lennox y Bell (2013) descartamos las revisiones sistemáticas, ya que en ellas no se podía llegar a establecer elementos de interacción. Finalmente localizamos un total de 40 artículos originales y dos investigadores de forma individual los revisaron.

La metodología seguida para la revisión de los artículos fue un análisis de contenido (Krippendorff, 1990) sobre las piezas originales. Para ello se elaboró una tabla ad hoc (Tabla 4) en la que incluimos todos los artículos, el foco de experiencia de paciente planteado en los mismos, a la vez que identificamos la terminología asociada con la comunicación vinculada con las relaciones entre pacientes, profesionales y organizaciones. Para la elaboración de la tabla, se partió en las primeras columnas los criterios de clasificación expuestos por Doyle, Lennox y Bell (2013) en su artículo original.

También incluimos el sistema de recopilación de evidencias de la experiencia de paciente para los artículos cuyo foco fue la comunicación, siguiendo los parámetros utilizados por los autores de la revisión sistemática, la existencia de evidencias entre la comunicación y la seguridad y efectividad clínicas.

\section{RESULTADOS}

¿En cuántos estudios se identifica la comunicación como foco de estudio en la experiencia de paciente? (P1)

${ }^{3}$ Términos de búsqueda que denotan la experiencia del paciente utilizados por Doyle, Lennox y Bell (2013): Patient-centred care; patient engagement; clinical interaction; patient-clinician; clinician-patient; patient-doctor; doctor-patient; physician- patient; patient-physician; patient-provider; interpersonal treatment; physician discussion; trust in physician; empathy; compassion; respect; responsiveness; patient preferences; shared decision-making; therapeutic alliance; participation in decisions; decision-making; autonomy; caring; kindness; dignity; honesty; participation; right to decide; physical comfort; involvement (of family, carers, friends); emotional support; continuity (of care); smooth transition; emotional support. 
Tras analizar los 40 artículos de la muestra observamos que el foco de estudio de experiencia de paciente se analiza en 69 contajes. El foco de experiencia de paciente hace referencia a los elementos vinculados a la experiencia del paciente (elementos no clínicos vinculados al estudio) sobre los que se ha centrado cada trabajo analizado.

En 17 de los casos, estos contajes son relacionados con el foco comunicación. Si a estos 17 casos añadimos todos aquellos elementos que precisan de forma directa para su razón de ser debemos añadir 8 casos más. Los datos agregados y de forma aislada se muestran en la tabla 1.

Tabla 1. Foco de experiencia de paciente

\begin{tabular}{l|l}
\hline \multicolumn{1}{c|}{ Foco de experiencia de paciente } & \multicolumn{1}{c}{ Contajes } \\
\hline $\begin{array}{l}\text { Comunicación, información, interacción, relación, } \\
\text { explicación }\end{array}$ & $25(17,2,3,2,1)$ \\
\hline $\begin{array}{l}\text { Atención, características del cuidado, coordinación, } \\
\text { continuidad, alta }\end{array}$ & $11(4,2,2,1,2)$ \\
\hline Satisfacción & 5 \\
\hline Confianza, fe & $5(3,2)$ \\
\hline Apoyo, alianza & $4(3,1)$ \\
\hline Experiencia del paciente en general & 3 \\
\hline Eventos adversos, errores & $3(2,1)$ \\
\hline $\begin{array}{l}\text { Participación toma de decisiones, preferencias del } \\
\text { paciente }\end{array}$ & $3(2,1)$ \\
\hline Empatía, cortesía, respeto y dignidad & $3(1,1,1)$ \\
\hline Calidad de los servicios & 2 \\
\hline Manejo del dolor & 1 \\
\hline Participación familiar & 1 \\
\hline Responsabilidad & 1 \\
\hline Competencia multicultural & 1 \\
\hline Accesibilidad & 1 \\
\hline
\end{tabular}

Fuente: Elaboración propia. El foco de experiencia del paciente hace referencia a todos aquellos elementos vinculados al estudio que no tienen carácter clínico. La tabla los presenta de forma agregada presentando primero los que obtienen frecuencias más elevadas. Los investigadores han considerado realizar agrupaciones temáticas para facilitar la lectura de los resultados.

\section{¿En cuántos estudios aparece la terminología asociada a la comunicación? (P2)}

De los 40 trabajos originales analizado, el término "comunicación" aparece en 29 casos, el término "información" en 25, "relación" en 21 e "interacción" en 21. Las menciones en función de si el foco de experiencia del trabajo es o no comunicación las encontramos en la tabla 2. Únicamente 3 de los trabajos no incluyen ni los términos "comunicación" ni "información", y 2 de éstos no incluyen ninguno de los términos de la búsqueda. Lógicamente, éstos tres trabajos no tienen cómo foco de experiencia de paciente la comunicación. 
La comunicación como elemento fundamental en la literatura especializada en experiencia de paciente (1989-2013)

Tabla 2. Terminología asociada a la comunicación y foco de experiencia de paciente

\begin{tabular}{|c|c|c|c|}
\hline & COM. & NO COM. & TOTAL \\
\hline Comunicación & 16 & 13 & 29 \\
\hline Información & 10 & 15 & 25 \\
\hline Promoción & 1 & 1 & 2 \\
\hline Relación & 11 & 11 & 22 \\
\hline Interacción & 10 & 11 & 21 \\
\hline Confianza & 1 & 2 & 3 \\
\hline Empatía & 1 & 1 & 2 \\
\hline
\end{tabular}

Fuente: Elaboración propia

¿Los sistemas de recopilación de datos de experiencia de paciente requieren interacción comunicativa? (P3)

Todas las evidencias sobre para la medición de la experiencia de paciente se recopilaron utilizando herramientas que requieren una interacción con el paciente. Ésta interacción fue en 25 casos utilizando encuestas, en 10 casos cuestionarios, en 6 casos revisando grabaciones de audio (en su mayoría de visitas) y en 2 casos entrevistas. Los datos entre los estudios centrados en la comunicación y los que no se muestran en la tabla 3. De las 10 encuestas utilizadas para la recopilación de datos en los estudios con foco en la comunicación 5 no únicamente estaban validadas, sino que formaban parte de los estándares de experiencia de paciente, igual que uno de los cuestionarios.

Tabla 3. Herramientas de recopilación de evidencias de experiencia de paciente

\begin{tabular}{|l|l|l|l|}
\hline & COM. & NO COM. & TOTAL \\
\hline Encuestas & 10 & & 25 \\
\hline Cuestionarios & 4 & 6 & 10 \\
\hline Entrevistas & 1 & 1 & 2 \\
\hline Cintas & 3 & 3 & 6 \\
\hline
\end{tabular}

Fuente: Elaboración propia

¿En cuántos casos se identifican variables de comunicación asociadas a variables de resultado clínico? (P4)

Por último, las asociaciones positivas entre la comunicación y resultados clínicos parecen consistentes en una amplia gama de áreas de enfermedad, diseños de estudio, entornos, grupos de población y medidas de resultado. Las asociaciones positivas encontradas superan "ninguna asociación" por 56 a 7. No se encontró ningún estudio en el que las "no asociaciones" fueran superiores a las asociaciones. En la tabla 4 se puede observar la distribución de asociaciones en la última columna. 
Entre las asociaciones positivas destacadas podemos mencionar la asociación entre competencias de empatía y multiculturalidad con satisfacción y multiculturalidad con adherencia, las interacciones con el equipo clínico y la comunicación médico paciente, la comunicación enfermera paciente, la escucha activa y mostrar atención, y entre las que no se ha encontrado asociación están las comunicaciones sobre medicación, las competencias de empatía con adherencia al tratamiento, la comunicación con la depresión o la comunicación con la edad de los pacientes.

Tabla 4. Análisis de los estudios seleccionados. Accesible en:

https://figshare.com/articles/dataset/Tabla 4 An lisis de los estudios seleccionado $\underline{\mathrm{s} / 14387519}$

\section{DISCUSIÓN}

El primer elemento de análisis de los distintos estudios fue el foco de experiencia de paciente, analizando los elementos no clínicos del estudio vinculados con la experiencia. De los cuatro pilares presentados por The Beryl Institute (2014), la interacción forma parte de la mayoría de los estudios, la atención continuada aparece en muy pocos, mientras que la cultura y la percepción están ausentes en todos los trabajos analizados.

A diferencia de los enfoques sobre la experiencia de paciente expuestos en el inicio de este artículo, recopilados por Wolf y otros (2014), donde los conceptos explícitos de "comunicación" e "información" eran muy minoritarios, el análisis de la selección bibliográfica propuesta por Doyle, Lennox y Bell (2013), más vinculada al ámbito clínico, apunta a un nuevo rol de la comunicación, mucho más protagonista, pudiendo considerarse uno de los elementos básicos que dotan de sentido a esta nueva especialidad sanitaria.

Concretamente, 17 de los 40 originales revisados en nuestra investigación incluían la comunicación en su foco de estudio vinculado a la experiencia de paciente, a los que se les podrían añadir otros elementos más, que directamente precisan de la comunicación, como la atención, la coordinación, la continuidad o el alta, la participación en la toma de decisiones, la participación familiar, la competencia multicultural, o la accesibilidad al sistema o al médico.

Esta lectura de los datos vinculados a los focos de estudio de cada original queda refutada cuando se observa que en únicamente 2 de los 40 analizados no aparece ninguna de las palabras vinculadas con la comunicación y se eleva a 29 el número de trabajos con el término exacto.

También es destacable que las herramientas de recopilación de datos para la evaluación de las distintas variables identificadas de la experiencia de paciente a partir del análisis de contenido precisan de interacciones diversas con los pacientes basadas en la comunicación con éstos. Debe proporcionarse información sobre las formas en que las experiencias de los pacientes con los proveedores han sido valiosas o problemáticas, 
y sobre cómo las organizaciones de atención de la salud pueden mejorar la atención del paciente (Agency for Healthcare Research and Quality, 2016). Debemos tener en cuenta también que, aunque la información cuantitativa sobre la experiencia del paciente ha dominado la medición y el informe de la calidad de la atención médica, la información cualitativa es cada vez más frecuente (Finucane y otros, 2018), algo que conlleva un aumento de interacciones vinculadas directamente con actos comunicativos.

Entendemos que el uso de la comunicación se presenta como imprescindible en los entornos sanitarios para referirse a la experiencia del paciente, convirtiéndose en un concepto transversal que transcurre entre autonomía, la elección, la confidencialidad, la dignidad, la atención inmediata y la calidad de los servicios básicos (Bleich, Ozaltin y Murray, 2009), a la vez que se sustenta en la visualización del paciente como participante activo, la receptividad de los servicios, el enfoque individualizado, la experiencia vivida, la continuidad de la atención y el apoyo (Staniszewska y otros, 2014). Por otra parte, las barreras de comunicación entre los proveedores de atención médica y los pacientes inmigrantes, por ejemplo, son extremadamente comunes y tienen un impacto y consecuencias importantes (Paternotte y otros, 2015).

La comunicación efectiva es esencial para mejorar el compromiso de los proveedores de salud y la experiencia del paciente (Barden A. y Giammarinaro, 2018). En esta línea, las 56 asociaciones positivas encontradas entre la comunicación y los resultados clínicos, sobre un total de 53, refuerzan la razón de ser de éste estudio y su carácter reivindicativo de espacio en las definiciones y abordajes de experiencia de paciente hasta hoy descuidados.

\section{CONCLUSIONES}

Se han identificado elementos de comunicación en la mayoría de los estudios analizados, no únicamente por su inclusión en el foco de estudio de muchos de ellos de forma directa o indirecta, sino porque el término comunicación y su campo semántico se encuentran en casi la totalidad de los mismos. Por otra parte, se observa que la comunicación es necesaria para utilizar cualquiera de las herramientas de medición de la experiencia de paciente, ya que estas mediciones conllevan interacciones con el paciente. Finalmente, podemos afirmar que la mayoría de las intervenciones en las que se precisa comunicación tienen asociaciones positivas con resultados clínicos, ya sean vinculados a la seguridad del paciente, el manejo de su condición o a la propia clínica de la patología.

\section{LIMITACIONES DEL ESTUDIO}

Esta revisión se plantea exclusivamente para los estudios clínicos previos a la definición de consenso de The Beryl Institute en 2014. Además, por rigor metodológico, los estudios incluidos se limitan a los encontrados en el estudio de referencia -el de Doyle, Lennox y Bell (2013)-, siendo un elemento que plantea sus limitaciones a la vez que refuerza la validez de la selección. Por otra parte, la heterogeneidad de las mediciones y de la terminología utilizada en los distintos estudios originales dificulta la 
agregación de los resultados. También debemos mencionar el sesgo de publicación, debido a que nosotros, por un lado, y los autores del estudio de referencia por otro, únicamente tenemos acceso a aquellos estudios que se han publicado y ello conlleva que solo disponemos de resultados de estudios con significación estadística.

\section{REFERENCIAS}

Agency for Healthcare Research and Quality (2016). Quality Improvement Rockville, MD: Agency for Healthcare Research and Quality.

Agoritsas, T., Bovier P. A. \& Perneger T. V. (2005). Patient reports of undesirable events during hospitalization. Journal of General Internal Medicinel, 20, 922-8. https://doi.org/10.1111//.1525-1497.2005.0225.x

Alamo, M. M., Moral, R. R. \& Pérula de Torres, L. A. (2002). Evaluation of a patientcentred approach in generalized musculoskeletal chronic pain/fibromyalgia patients in primary care. Patient Education and Counseling, 48, 23-31. https://doi.org/10.1016/s0738-3991(02)00095-2

Arbuthnott, A. \& Sharpe, D. (2009). The effect of physician-patient collaboration on patient adherence in non-psychiatric medicine. Patient Education and Counseling, 77, 60-7. https://doi.org/10.1016/j.pec.2009.03.022

Barden, A. \& Giammarinaro, N. (2018). Effectiveness of the communication model, C.O.N.N.E.C.T., on patient experience and employee engagement: A prospective study. Patient Experience Journal, 5(3). https://doi.org/10.35680/2372-0247.1314

Beck, R. S., Daughtridge, R. \& Sloane, P. D. (2002). Physician-patient communication in the primary care office: a systematic review. Journal of the American Board of Family Medicine,15, 25-38. https://pubmed.ncbi.nlm.nih.gov/11841136/

Begg, C. \& Berlin, J. (1988). Publication bias: a problem in interpreting medical data. Journal of the Royal Statistical Society, 151. https://doi.org/10.2307/2982993

Berwick, D. M. (2009). What 'patient-centered' should mean: confessions of an extremist. Health Affairs, 28, 555-65. https://doi.org/10.1377/hlthaff.28.4.w555

Black, N. \& Jenkinson, C. (2009). Measuring patients experiences and outcomes. British Medical Journal, 339, 202-5. https://doi.org/10.1136/bmj.b2495

Blasi, Z. D., Harkness, E., Ernst, E. et al (2001). Influence of context effects on health outcomes: a systematic review. Lancet, 357, 757-62. https://doi.org/10.1016/S01406736(00)04169-6 
Bleich, S. N., Ozaltin, E. \& Murray, C. J. L. (2009). How does satisfaction with the healthcare system relate to patient experience? Bulletin of the World Health Organization, 87(4), 271-278. https://doi.org/10.2471/blt.07.050401

Bowling, A., Rowe, G., Lambert, N. et al. (2012). The measurement of patients' expectations for health care: a review and psychometric testing of a measure of patients' expectations. Health Technology Assessment (Winchester, England), 16(30), i-xii, 1-509. https://doi.org/10.3310/hta16300

Burgers, J. S., Voerman, G. E., Grol, R. et al. (2010). Quality and coordination of care for patients with multiple conditions: results from an international survey of patient experience. Evaluation \& the Health Professions, 33, 343-64. https://doi.org/10.1177/0163278710375695

Cabana, M. \& Jee, S. (2004) Does continuity of care improve patient outcomes? Journal of Family Practices, 53. https://pubmed.ncbi.nlm.nih.gov/15581440/

Carcaise-Edinboro, P. \& Bradley C. J. (2008). Influence of patient-provider communication on colorectal cancer screening. Medical Care, 46, 738-45. https://doi.org/10.1097/MLR.0b013e318178935a

Chang, J. T., Hays, R. D., Shekelle, P. G. et al. (2006). Patients' global ratings of their health care are not associated with the technical quality of their care. Annals of Internal Medicine, 145, 635-6. https://doi.org/10.7326/0003-4819-144-9-200605020-00010

Clark, N. M., Cabana, M. D., Nan, B. et al. (2008). The clinician-patient partnership paradigm: outcomes associated with physician communication behavior. Clinical Pediatrics, 47,49-57. https://doi.org/10.1177/0009922807305650

Coulter, A. (2011). Engaging patients in healthcare. Maidenhead, Berkshire: Open University Press.

Darzi, A. (2008). High quality care for all. NHS Next Stage Review Final Report. Department of Health. https://www.gov.uk/government/publications/high-quality-carefor-all-nhs-next-stage-review-final-report

Department of Health (2010). Liberating the NHS: transparency in outcomes. A framework for the NHS: Department of Health.

Department of Health (2008). Using the Commissioning for Quality and Innovation (CQUIN) payment framework.

Doyle, C., Lennox, L., \& Bell, D. (2013). A systematic review of evidence on the links between patient experience and clinical safety and effectiveness. BMJ Open, 3(1), 118. http://dx.doi.org/10.1136/bmjopen-2012-001570 
La comunicación como elemento fundamental en la literatura especializada en experiencia de paciente (1989-2013)

Drotar, D. (2009). Physician behavior in the care of pediatric chronic illness: association with health outcomes and treatment adherence. Journal of Developmental and Behavioral Pediatrics, 30, 246-54. https://doi.org/10.1097/DBP.0b013e3181a7ed42

Fan, V. S., Reiber, G. E., Diehr, P. et al. (2005). Functional status and patient satisfaction. Journal of General Internal Medicine, 20, 452-9. https://doi.org/10.1111/j.15251497.2005.40057.x

Feirn, A., Betts D. \& Tribble, T. (2009). The patient experience: strategies and approaches for providers to achieve and maintain a competitive advantage. Deloitte LLP's Health Sciences Practice White Paper.

Finucane, M. L., Martino, S. C., Parker, A. M. Et al (2018). A framework for conceptualizing how narratives from health-care consumers might improve or impede the use of information about provider quality, Patient Experience Journal. 5(1). https://doi.org/10.35680/2372-0247.1229

Flocke, S. A., Stange, K. C. y Zyzanski, S. J. (1998). The association of attributes of primary care with the delivery of clinical preventive services. Medical Care, 36, 21-30. https://doi.org/10.1097/00005650-199808001-00004

Frampton, S. B. (2012). Healthcare and the patient experience: harmonizing care and environment. HERD, 5(2), 3-6. https://doi.org/10.1177/193758671200500201

Fremont, A., Cleary, P., Hargraves, J. et al. (2001). Patient-centered processes of care and long-term outcomes of myocardial infarction. Journal of General Internal Medicine, 16, 800-8. https://doi.org/10.1111/.1525-1497.2001.10102.x

Fuertes, J., Boylan, L. \& Fontanella, J. (2009). Behavioral indices in medical care outcome: the working alliance, Adherence, and related factors. Journal of General Internal Medicine, 24, 80-5. https://doi.org/10.1007/s11606-008-0841-4

Glickman, S. W., Boulding, W., Manary, M. et al. (2010). Patient satisfaction and its relationship with clinical quality and inpatient mortality in acute myocardial infarction. Circulation: Cardiovascular Quality and Outcomes, 3, 188-95. https://doi.org/10.1161/CIRCOUTCOMES.109.900597

Greenhalgh, T. \& Peacock, R. (2005). Effectiveness and efficiency of search methods in systematic reviews of complex evidence: audit of primary sources. British Medical Journal, 331, 1064-65. https://doi.org/10.1136/bmj.38636.593461.68

Hall, A. M., Ferreira, P. H., Maher, C. G. et al. (2010). The influence of the therapist-patient relationship on treatment outcome in physical rehabilitation: a systematic review. Physical Therapy, 90, 1099-110. https://doi.org/10.2522/ptj.20090245 
Hall, J. A., Roter, D. L. \& Katz, N. R. (1988). Meta-analysis of correlates of provider behavior in medical encounters. Medical Care, 26, 657-75. https://doi.org/10.1097/00005650-198807000-00002

Haynes, R. B., Ackloo, E., Sahota, N. et al. (2008). Interventions for enhancing medication adherence. The Cochrane Database of Systematic Reviews, 2(37) https://doi.org/10.1002/14651858.CD000011.pub4

Heisler, M., Bouknight, R. R., Hayward, R. A. et al. (2002). The relative importance of physician communication, participatory decision making, and patient understanding in diabetes self-management. Journal of General Internal Medicine, 17, 243-52. https://doi.org/10.1046//.1525-1497.2002.10905.x

Hewitson, P., Skew, A., Graham, C., et al. (2014). People with limiting long-term conditions report poorer experiences and more problems with hospital care. BMC Health Services Research, 14. https://doi.org/10.1186/1472-6963-14-33

Hsiao, C. J. \& Boult, C. (2008). Effects of quality on outcomes in primary care: a review of the literature. American Journal of Medical Quality, 23, 302-10. https://doi.org/10.1177/1062860608315643

Iles, V. \& Vaughan, S. J. (2009). Working in health care could be one of the most satisfying jobs in the world-why doesn't it feel like that?

Institute of Medicine (2011). Crossing the quality chasm: a new health system for the 21 st century. Washington, DC: National Academy Press.

Isaac, T., Zaslavsky, A. M., Cleary P. D. et al. (2010). The relationship between patients' perception of care and measures of hospital quality and safety. Health Services Research Journal, 45, 1024-40. https://doi.org/10.1111/j.1475-6773.2010.01122.x

Jackson, C. A., Clatworthy, J., Robinson, A. et al. (2010). Factors associated with nonadherence to oral medication for inflammatory bowel disease: a systematic review. The American Journal of Gastroenterology, 105, 525-39. https://doi.org/10.1038/ajg.2009.685

Jackson, J. L., Chamberlin, J. \& Kroenke, K. (2001). Predictors of patient satisfaction. Social Science\&Medicine, 52, 609-20. https://doi.org/10.1016/s0277-9536(00)00164$\underline{7}$

Jha, A. K., Orav, E. J., Zheng, J. et al. (2008). Patients' perception of hospital care in the United States. The New England Journal of Medicine, 359, 1921-31. https://www.nejm.org/doi/full/10.1056/nejmsa0804116 
Kahn, K. L., Schneider, E. C., Malin, J. L. et al. (2007). Patient centered experiences in breast cancer: predicting long-term adherence to tamoxifen use. Medical Care, 45, 431-9. https://doi.org/10.1097/01.mlr.0000257193.10760.7f

Kaplan, S. H., Greenfield, S. \& Ware, J. E. (1989). Assessing the effects of physicianpatient interactions on the outcomes of chronic disease. Medical Care, 27(3 Suppl): S110-27. https://doi.org/10.1097/00005650-198903001-00010

Kennedy, A., Nelson, E., Reeves, D. et al. (2003). A randomised controlled trial to assess the impact of a package comprising a patient-orientated, evidence-based self-help guidebook and patient-centred consultations on disease management and satisfaction in inflammatory bowel disease. Health Technology Assessment (Winchester, England), 7 (iii), 1-113. https://doi.org/10.3310/hta7280

Kinnersley, P., Stott, N., Peters, T. J. et al. (1999). The patient-centredness of consultations and outcome in primary care. British Journal of General Practice, 49, 711-16. PMC1313498

Krippendorff, K. (1990). Metodología del análisis de contenido: teoría y práctica. Barcelona: Planeta.

Lee, Y-Y. \& Lin, J. L. (2010). Do patient autonomy preferences matter? Linking patientcentered care to patient-physician relationships and health outcomes. Social Science\&Medicine, 71, 1811-18. https://doi.org/10.1016/j.socscimed.2010.08.008

Lee, Y-Y. \& Lin, J. L. (2009). The effects of trust in physician on self-efficacy, adherence and diabetes outcomes. Social Science\&Medicine, 68, 1060-8. https://doi.org/10.1016//.socscimed.2008.12.033

Levinson, W., Roter, D. L., Mullooly, J. P. et al. (1997). Physician-patient communication: the relationship with malpractice claims among primary care physicians and surgeons. Journal of the American Medical Association, 277, 553-9. https://doi.org/10.1001/jama.277.7.553

Lewis, E. T., Combs, A. y Trafton, J. A. (2010). Reasons for under-use of prescribed opioid medications by patients in pain. Pain Medicine, 11, 861-71. https://doi.org/10.1111//.1526-4637.2010.00868.x

Little, P., Everitt, H., Williamson, I. et al. (2001). Observational study of effect of patient centredness and positive approach on outcomes of general practice consultations. British Medical Journal, 323, 908-11. https://doi.org/10.1136/bmj.323.7318.908

López, L., Weissman, J. S., Schneider, E. C. et al. (2019). Disclosure of hospital adverse events and its association with patients' ratings of the quality of care. Archives of Internal Medicine, 169, 1888-94. https://doi.org/10.1001/archinternmed.2009.387 
Meterko, M., Wright, S., Lin, H. et al. (2010). Mortality among patients with acute myocardial infarction: the influences of patient-centered care and evidence-based medicine. Health Services Research Journal, 45 (5p1), 1188-204. https://doi.org/10.1111/j.1475-6773.2010.01138.x

Mumford, E., Schlesinger, H. J. y Glass, G. V. (1982). The effect of psychological intervention on recovery from surgery and heart attacks: an analysis of the literature. American Public Health Association, 72, 141-51. https://doi.org/10.2105/ajph.72.2.141

Needham, B. R. (2012). The truth about patient experience: what we can learn from other industries, and how three ps can improve health outcomes, strengthen brands, and delight customers. Journal of Healthcare Management / American College of Healthcare Executives, 57(312), 255-63. https://pubmed.ncbi.nlm.nih.gov/22905604/

NICE (2011). Patient experience in adult NHS services: improving the experience of care for people using adult NHS services. Manchester: NICE. https://pubmed.ncbi.nlm.nih.gov/23285499/

O'Connor, A. M., Bennett, C. L., Stacey, D. et al. (2009). Decision aids for people facing health treatment or screening decisions. Cochrane database of Systematic Reviews, 3, CD001431. https://doi.org/10.1002/14651858.CD001431.pub5

O'Malley, A. S., Sheppard, V. B., Schwartz, M. et al. (2004). The role of trust in use of preventive services among low-income African-American women. Preventive Medicine, 38, 777-85. https://doi.org/10.1016/j.ypmed.2004.01.018

Paternotte, E., Van Dulmen, S., Van der Lee, N. et al. (2015). Factors influencing intercultural doctor-patient communication: A realist review. Patient education and counseling, 98(4), 420- 445. https://doi.org/10.1016/j.pec.2014.11.018

Pemberton, S. \& Richardson, H. A. (2013). Vision of the future for patient experience. Nursing Times, 109, 33- 34. https://www.nursingtimes.net/clinical-archive/patientsafety/a-vision-of-the-future-for-patient-experience-16-08-2013/

Picker Institute (2008). Patient experience surveys: the rationale Picker Institute Europe, Oxford.

Plomondon, M., Magid, D., Masoudi, F. et al. (2008). Association between angina and treatment satisfaction after myocardial infarction. Journal of General Internal Medicine, 23, 1-6. https://doi.org/10.1007/s11606-007-0430-y

Raiz, L. R., Kilty, K. M., Henry, M. L. et al. (1999). Medication compliance following renal transplantation. Transplantation, 68, 51-5. https://doi.org/10.1097/00007890$\underline{199907150-00010}$ 
Rao, M., Clarke, A., Sanderson, C. et al. (2006). Patients' own assessments of quality of primary care compared with objective records-based measures of technical quality of care: cross sectional study. British Medical Journal, 333, 19-22. https://doi.org/10.1136/bmj.38874.499167.7C

Rathert, C., Huddleston, N. \& Pak, Y. (2011). Acute care patients discuss the patient role in patient safety. Health Care Management Review, 36, 134-44. https://doi.org/10.1097/HMR.0b013e318208cd31

Richards, H. L., Fortune, D. G. \& Griffiths, C. E. M. (2006). Adherence to treatment in patients with psoriasis. Journal of the European Academy of Dermatology and Venereology, 20, 370-9. https://doi.org/10.1111/j.1468-3083.2006.01565.x

Riley, D. L., Stewart, D. E. \& Grace, S. L. (2007). Continuity of cardiac care: cardiac rehabilitation participation and other correlates. International Journal of Cardiology, 119, 326-33. https://doi.org/10.1016/j.ijcard.2006.07.158

Robert Wood Johnson Foundation. Good for Health, good for business: the case for measuring patient experience of care. The Center for Health Care Quality at the George Washington University Medical Center, Washington DC. http://forces4quality.org/af4q/download-document/3212/ResourceCase\%20for\%20Patient\%20Experience branded2.pdf

Robison, J. (2010). What is the "patient experience"? Gallup Management Journal Online. https://news.gallup.com/businessjournal/143258/patient-experience.aspx

Roth, G. (2014). The Importance of Communication and Coordination for a Positive Patient Experience. En: Patient Experience Transformation: Engaged Patients, Measurable Standards An Independent Health Leaders Media Survey. Team Health.

Safran, D. G., Miller, W. \& Beckman, H. (2005). Organizational dimensions of relationchipcentred care. Journal of General Internal Medicine, 21, S9-15. https://doi.org/10.1111//.1525-1497.2006.00303.x

Safran, D. G., Taira, D. A., Rogers, W. H. et al. (1998). Linking primary care performance to outcomes of care. Journal of Family Practice, 47, 213-20. https://pubmed.ncbi.nlm.nih.gov/9752374/

Salomon, M. (2014). "9 Patient Experience And Satisfaction Secrets For Hospitals And Healthcare". $\quad$ https://www.forbes.com/sites/micahsolomon/2014/09/08/7-thingshospitals-and-healthcare-professionals-get-wrong-about-the-patient-experience-andpatient-satisfaction/?sh=2bc381dd7e1d

Shale, S. (2013). Patient experience as an indicator of clinical quality in emergency care. Clinical Governance: An International Journal. https://doi.org/10.1108/CGlJ-03-2012$\underline{0008}$ 
Sans-Corrales, M., Pujol-Ribera, E., Genís-Badia, J. et al. (2006). Family medicine attributes related to satisfaction, health and costs. Family Practice, 23, 308-16. https://doi.org/10.1093/fampra/cmi112

Saultz, J. W. \& Lochner, J. (2005). Interpersonal continuity of care and care outcomes: a critical review. The Annals of Family Medicine, 3, 159-66. https://doi.org/10.1370/afm.285

Schneider, E. C., Zaslavsky, A. M., Landon, B. E. et al. (2001). National quality monitoring of medicare health plans: the relationship between enrollees' reports and the quality of clinical care. Medical Care, 39, 1313-25. https://doi.org/10.1097/00005650$\underline{\text { 200112000-00007 }}$

Schoenthaler, A., Chaplin, W. F., Allegrante, J. P. et al. (2009). Provider communication effects medication adherence in hypertensive African Americans. Patient Education and Counseling, 75, 185-91. https://doi.org/10.1016/.j.pec.2008.09.018

Sequist, T. D., Schneider, E. C., Anastario, M. et al. (2008). Quality monitoring of physicians: Linking patients' experiences of care to clinical quality and outcomes. Journal of General Internal Medicine, 23(11), 1784-1790. https://doi.org/10.1007/s11606-008-0760-4

Slatore, C. G., Cecere, L. M., Reinke, L. F. et al. (2010). Patient-clinician communication: Associations with important health outcomes among veterans with COPD. Chest, 138(3), 628-634. https://doi.org/10.1378/chest.09-2328

Solberg, LI., Asche, S. E., Averbeck, B. M. et al. (2008). Can patient safety be measured by surveys of patient experiences. Joint Commission Journal on Quality and Patient Safety, 34, 266-74. https://doi.org/10.1016/s1553-7250(08)34033-1

Staniszewska, S. \& Bullock, I. (2012). Can we help patients have a better experience? Implementing NICE guidance on patient experience. Evidence-Based Nursing, 15(4), 99. https://doi.org/10.1136/eb-2012-100988

Staniszewska, S., Boardman, F., Gunn, L. et al. (2014). The warwick patient experiences framework: Patient-based evidence in clinical guidelines. International Journal for Quality in Health Care, 26(2), 151-157. http://doi.org/10.1093/intahc/mzu003

Stempniak, M. (2013). The patient experience. Taking it to the next level. Hospitals \& Health Networks / AHA, 87(4), 41-47. https://pubmed.ncbi.nlm.nih.gov/23700758/

Stevenson, F. A., Cox, K., Britten, N. et al. (2004). A systematic review of the research on communication between patients and health care professionals about medicines: the consequences for concordance. Health Expectations, 7, 235-45. https://doi.org/10.1111//.1369-7625.2004.00281.x 
Stewart, M., Brown, J., Donner, A. et al. (2000). The impact of patient-centered care on outcomes. Journal of Family Practices, 49, 796-804. https://pubmed.ncbi.nlm.nih.gov/11032203/

Stewart, M. A. (1995). Effective physician-patient communication and health outcomes: a review. Canadian Medical Association Journal, 152, 1423-33. https://pubmed.ncbi.nlm.nih.gov/7728691/

Street, R. L., Makoul, G., Arora, N. K. et al. (2009). How does communication heal? Pathways linking clinician-patient communication to health outcomes. Patient Education and Counseling, 74, 295-301. https://doi.org/10.1016/j.pec.2008.11.015

The Beryl Institute (2014). Defining Patient Experience. https://www.theberylinstitute.org/page/DefiningPatientExp

Thom, D. H., Hall, M. A. \& Pawlson, L. G. (2004). Measuring patients' trust in physicians when assessing quality of care. Health Affairs, 23, 124-32. https://doi.org/10.1377/hlthaff.23.4.124

Vincent, C. (2003). Understanding and responding to adverse events. The New England Journal of Medicine, 348, 1051-6. https://www.nejm.org/doi/pdf/10.1056/NEJMhpr020760

Vincent, C. A. \& Coulter, A. (2002). Patient safety: what about the patient? Quality and Safety in Health Care, 11, 76-80. https://doi.org/10.1136/ahc.11.1.76

Weingart, S. N., Pagovich, O., Sands, D. Z. et al. (2005). What can hospitalized patients tell us about adverse events? Learning from patient-reported incidents. Journal of General Internal Medicine, 20, 830-6. https://doi.org/10.1111/j.1525-1497.2005.0180.x

Weiss, M., Tyink, S. (2009). Creating sustainable ideal patient experience cultures. MEDSURG, 18(4), 249-252. https://pubmed.ncbi.nlm.nih.gov/20552854/

Weissman, J. S., Schneider, E. C., Weingart, S. N. et al. (2008). Comparing patientreported hospital adverse events with medical record review: do patients know something that hospitals do not? Annals of Internal Medicine, 149, 100-8. https://doi.org/10.7326/0003-4819-149-2-200807150-00006

Wolf, J., Niederhauser, V., Marshburn, D., La Vela, S. (2014). Defining patient experience. Patient Experience Journal, 1(1), 7-19. https://pxjournal.org/journal/vol1/iss1/3/

Wolf, J. A. (2017). A report on The Beryl Institute benchmarking study. The state of patient experience 2017: a return to purpose. The Beryl Institute. https://www.theberylinstitute.org/store/ViewProduct.aspx?id=13390491 
Zolnierek, H. K. B. \& DiMatteo, M. R. (2009). Physician communication and patient adherence to treatment: a meta-analysis. Medical Care, 47, 826-34. https://doi.org/10.1097/MLR.0b013e31819a5acc

\section{AUTORES}

\section{Dr. Guillem Marca-Francès}

Profesor del Departamento de Comunicación, Facultad de Empresa y Comunicación, Universitat de Vic-Universitat Central de Catalunya (UVic-UCC). Miembro del grupo de investigación Learning, Media \& Social Interactions (2017SGR379). Especializado en comunicación de la salud y en relaciones públicas.

ORCID ID: https://orcid.org/0000-0002-1586-8625

Google Scholar ID: https://scholar.google.com/citations?hl=ca\&user=v6hT7SIAAAAJ

\section{Dr. Marc Compte-Pujol}

Profesor del Departamento de Comunicación, Facultad de Empresa y Comunicación, Universitat de Vic-Universitat Central de Catalunya (UVic-UCC). Miembro del grupo de investigación Learning, Media \& Social Interactions (2017SGR379). Especializado en comunicación de la salud y en relaciones públicas.

ORCID ID: https://orcid.org/0000-0002-6694-2485

Google Scholar ID: https://scholar.google.com/citations?hl=ca\&user=ikmknx8AAAAJ

\section{Dr. Jesica Ana Menéndez-Signorini}

Profesora del Departamento de Comunicación, Facultad de Empresa y Comunicación, Universitat de Vic-Universitat Central de Catalunya (UVic-UCC). Miembro del grupo de investigación Learning, Media \& Social Interactions (2017SGR379). Especializada en comunicación de la salud y en RSC.

ORCID ID: https://orcid.org/0000-0002-4833-5956

Google Scholar ID: https://scholar.google.com/citations?hl=ca\&user=dPwa1ZsAAAAJ

\section{Dr. Joan Frigola-Reig}

Profesor del Departamento de Comunicación, Facultad de Empresa y Comunicación, Universitat de Vic-Universitat Central de Catalunya (UVic-UCC). Miembro del grupo de investigación Learning, Media \& Social Interactions (2017SGR379). Especializado en comunicación de la salud y formatos audiovisuales.

ORCID ID: https://orcid.org/0000-0003-4195-1104 